\title{
POLITICA POPULAR EN TIEMPOS DE ECONOMÍAS POSTINDUSTRIALES: TRABAJO TERRITORIAL Y ECONOMÍA MORAL EN LA ARGENTINA RECIENTE
}

\section{POPULAR POLITICS IN POST-INDUSTRIAL ECONOMIES TIMES: TERRITORIAL WORK AND MORAL ECONOMY IN CURRENT ARGENTINA}

Gabriel Vommaro*

Introducción: las clases populares y la política. Cambios morfológicos, intervenciones estatales y nuevos problemas analíticos

Las transformaciones de los vínculos de las clases populares con el trabajo asalariado formal han sido estudiadas en Argentina (MAURIZIO et al., 2008) y en Latinoamérica (KESSLER; DI VIRGILIO, 2008), y fueron objeto de estudios ya clásicos sobre los países capitalistas centrales (CASTEL, 1995; RIFKIN, 1995; ROSANVALLON, 1995). En el caso argentino podemos resumir estas transformaciones a los procesos de empobrecimiento, de desalarización y de informalización de esos sectores, producidos por la implementación de políticas neoliberales que acompañaron la desindustrialización capitalista, a los cuales le siguieron, primeramente, una serie de respuestas estatales en términos de políticas sociales de lucha contra la pobreza, y, más tarde, de políticas de inclusión. El modo de aplicación de estas políticas, con sus diferentes enfoques desde los años noventa del siglo XX hasta la actualidad, así como el tipo de entramado socio-cultural en que se insertaron, son factores centrales en la producción de nuevos vínculos de las clases populares con la política. El objeto de este trabajo es proponer algunas herramientas analíticas para analizar esos vínculos. Para ello, movilizamos materiales empíricos recogidos en trabajos de campo realizados entre 2006 y

\footnotetext{
* Investigador de CONICET, Investigador-docente de la Universidad Nacional de General Sarmiento - Área de Política, Instituto del Desarrollo Humano - (UNGS/Buenos Aires/AR), doctor en sociología por la Ecole des Hautes Etudes en Sciences Sociales (CSE). gvommaro@ungs.edu.ar
} 
2016 en dos barrios populares ubicados en diferentes distritos de Argentina ${ }^{1}$.

En ese país, el entramado socio-cultural de las clases populares estaba dominado, hasta los años ochenta, por el activismo social barrial de los partidos políticos, en especial del peronismo, la fuerza más arraigada en el mundo popular ${ }^{2}$, y, en segundo lugar, por la presencia de actores del catolicismo social. Los nuevos modos de intervención del Estado a nivel local, producidos a partir de las políticas sociales de combate a la pobreza, favorecieron la entrada de otros actores, emergentes de procesos con otras temporalidades - el ingreso de fuerzas políticas en los barrios que disputaron la hegemonía peronista, la aparición de activistas de raíz autonomista, la reconversión de líderes sindicales en líderes sociales barriales (MARTUCCELLI; SVAMPA, 1997). Estos actores intervinieron en la implementación de los programas de asistencia de diferente tipo; así, entre otros, movimientos sociales territoriales (MERKLEN, 2005; MANZANO, 2013; QUIROS, 2012) y grupos evangélicos (SEMAN, 2006) ampliaron la lista de participantes de la política a nivel barrial. Esta intervención fue posible, en buena medida, porque las "políticas de compensación" de los efectos sociales de las reformas neoliberales fueron implementadas en consonancia con la filosofía de la focalización, la descentralización y el empoderamiento de la sociedad civil que promovían los organismos multilaterales como el Banco Mundial y el BID³. La sociedad civil fomentada desde el Estado tomaba formas inesperadas en el territorio: los dirigentes políticos barriales del peronismo, así como de los nuevos movimientos territoriales, creaban asociaciones civiles para volverse espacios de "contraprestación"4 de los programas sociales -de asistencia a comedores, de transferencia

1. Las observaciones fueron realizadas en dos etapas. La primera, entre los años 2006 y 2007, en un barrio popular de la periferia de la ciudad de Santiago del Estero, en el noroeste argentino. La segunda, entre 2014 y 2016, en un asentamiento ubicado en el barrio de Villa Soldati, en el sur de la Ciudad de Buenos Aires. Los programas sociales que identificamos en el primer trabajo de campo dejaron de existir, y en su lugar surgieron otros. Como veremos, la centralidad del trabajo político y social en la construcción de vínculos políticos barriales se mantuvo como una constante de ambos periodos.

2. Desde sus origenes, el peronismo construye su arraigo en el mundo popular por una doble vía: la de la integración de los trabajadores formales en las organizaciones sindicales fuertemente vinculadas a él, y la de la movilización de sectores informales, tanto a través de asociaciones barriales incorporadas al movimiento peronista como de políticas públicas de asistencia, cuyo emblema fue la Fundación Eva Perón. Sobre el vínculo del peronismo con el mundo obrero, cf. (DEL CAMPO, 1983; JAMES, 1990). Sobre la asociatividad barrial ligada al primer peronismo, cf. (ACHA, 2004). Sobre la Fundación Eva Perón, cf. (PLOTKIN, 1993). Más recientemente, Steven Levitsky (2003) estudió el proceso de debilitamiento del peso de los sindicatos en el movimiento peronista - de la mano de la crisis del mundo industrial y, más coyunturalmente, de los efectos de la derrota electoral de 1983 -, y el auge de las redes territoriales manejadas por políticos profesionales, que el autor llama redes clientelares.

3. Sobre los rasgos principales de las políticas sociales en Argentina desde los años ochenta, cf. (CORTÉS; KESSLER, 2013).

4. La noción de contraprestación refiere a la contraparte que los beneficiarios de los programas sociales deben realizar para cumplir con los requisitos de acceso a esos bienes. Desde mediados de los años noventa, buena parte de esos programas exige alguna forma de trabajo comunitario, realizado en los espacios de sociabilidad política barriales, o organizado por esos espacios en el ámbito local (pequeños arreglos de calles, veredas, etc.). 
condicionada, de distribución de alimentos y protección de madres y embarazadas, etc.- y de este modo lograban mantener el vínculo con sus bases y hasta ampliar su capacidad de movilización barrial.

$\mathrm{Al}$ distribuir bienes entre los pobres, estos actores fueron definidos por académicos y otros observadores de la política como protagonistas de lógicas clientelares. El peronismo, en esos análisis, pasaba de ser un partido de base sindical a uno de base clientelar (LEVITSKY, 2003). Por esta y por otras vías - como los debates sobre la "calidad de la democracia" (DIAMOND; MORLINO, 2004) -, el enfoque del clientelismo se volvió dominante para pensar la nueva politicidad popular. Como hemos mostrado en otra parte (VOMMARO, 2010; COMBES; VOMMARO, 2015), éste devino, asimismo, una categoría nativa de descalificación política, movilizada tanto en las querellas morales protagonizadas por los actores de la política barrial como en las miradas de los observadores interesados en "la política de los pobres": profesionales del comentario político, por usar los términos de Offerlé (1988), actores eclesiales, miembros de ONG y fundaciones expertas...

Los estudios de tipo etnográfico realizados en Argentina a partir de fines de los años noventa y en especial luego de la multiplicación de programas sociales dirigidos al mundo popular - y de los actores encargados de su "gestión" -, mostraron que la complejidad de los vínculos políticos, construidos en torno a las políticas sociales trascendian los intercambios clientelares. Situados en las configuraciones locales barriales, desarmaron las dicotomías entre dirigentes barriales heroicos - en especial, los llamados "piqueteros" ${ }^{5}$, y agentes de la dominación - los "punteros" peronistas (referentes barriales)-, (QUIRÓS, 2011; VOMMARO, 2007) y dieron cuenta de que todos ellos formaban parte de un entramado complejo de competencia en el que emprendedores territoriales se ocupaban, por un lado, de la movilización de las clases populares y, por el otro, de la distribución de bienes de origen público destinados al desarrollo de la vida de las familias de esas clases. En ambos los casos, mostraron que en torno a ellos se fundaban espacios locales de sociabilidad política (VOMMARO, 2007) en los que se desarrollaba la politicidad popular (MERKLEN, 2005) - es decir la sociabilidad política cotidiana de esas clases, al menos de sus fracciones desalarizadas -, en la que se establecen vínculos de más o menos largo plazo que incluyen acompañamientos políticos y formas de trabajo social y comunitario (MANZANO, 2013; QUIRÓS, 2011) hechas de conflictos y negociaciones. Por último, demostraron, en la línea de los trabajos de Auyero (1997, 2001) y Martuccelli e Svampa (1997), que era en la temporalidad de estas relaciones que debían insertarse los intercambios políticos habitualmente estudiados en términos de relaciones clientelares espasmódicas, y que por tanto eran objeto de una regulación política y moral que no podía reducirse a transacciones esporádicas y puramente instrumentales (COMBES; VOMMARO, 2015; FERRAUDI CURTO, 2014; VOMMARO; QUIRÓS, 2011) $)^{6}$.

5. Sobre la historia y las características del movimiento piquetero, cf. (SVAMPA; PEREYRA, 2003).

6. Con puntos de vista afines a los desarrollados por Briquet (1997) para el caso de las relaciones políticas personalizadas en Córcega y por Bezerra (1999) para el de los servicios que los parlamentarios brasileños ofrecen a sus bases electorales. 
En línea con esos hallazgos, en este trabajo quisiéramos argumentar que las dinámicas políticas cotidianas de las fracciones desalarizadas de las clases populares pueden ser aprehendidas a partir de dos tipos de herramientas conceptuales. Por un lado, si utilizamos un modo de pensar la relación entre Estado y sociedad civil que, a priori, no presupone los límites de una y otra entidad y, en continuidad con la tradición de estudios de la burocracia de calle (LIPSKY, 1980; DUBOIS, 1999), estudia los lazos porosos entre ambas ${ }^{7}$. Esto en condiciones en que los actores sociales barriales funcionan como gestores de políticas sociales $\mathrm{y}$ mediadores entre las agencias estatales centrales y los vecinos; y en que el Estado fomenta su manifestación empírica en y a través de esos agentes locales ${ }^{8}$. Nuestro modo de proceder, sostenemos, permite pensar las regulaciones de la actividad política en el entrecruzamiento entre lógicas estatales y lógicas socio-políticas barriales, y reconstruir las nociones de derecho movilizadas por las clases populares en tiempos de capitalismo post-industrial. Por el otro, y en relación al punto anterior, las nociones de economía moral (THOMPSON, 1995) y de arreglos morales (VOMMARO; QUIRÓS, 2011; COMBES; VOMMARO, 2015; VOMMARO, 2016) permiten aprehender las regulaciones morales de los intercambios y vínculos políticos personalizados en los que intervienen las clases populares informales, que organizan los principios de justicia con los que se juzgan la producción y la circulación de bienes - en lo fundamental de origen público -, en la era de las políticas sociales de transferencia condicionada de recursos. El artículo está organizado en tres partes, que discuten sucesivamente ambas problemáticas. En primer lugar, las formas de porosidad y superposición entre el Estado y la sociedad civil en el mundo popular. En segundo lugar, las actividades que realizan las clases populares informales en esos espacios socio-estatales - a nivel barrial y en virtud de su carácter de beneficiarios de programas sociales -, son concebidas como formas de "trabajo". En tercer lugar, la economía moral del bienestar de los pobres que surge de esa configuración. Al final, hacemos algunos comentarios sobre los aportes que puede realizar esta perspectiva para un conocimiento más acabado de la politicidad popular.

\section{Las políticas sociales, la sociedad civil y la porosidad socio-política del Estado}

Desde los años noventa del siglo pasado, las agencias del Estado argentino se propusieron co-gestionar las políticas sociales - programas de transferencia condicionada, programas de ayuda alimentaria, etc. -, con la sociedad civil. El imperativo del "empo-

7. Sobre este punto, es necesario referir también a los estudios etnográficos sobre el funcionamiento cotidiano del Estado realizados por algunos antropólogos post-coloniales, que han identificado estos "límites borrosos" entre las agencias estatales y la sociedad civil. Cf., por ejemplo, (GUPTA, 1995). En una línea diferente pero también alternativa a las teorías de los límites precisos del Estado, la sociología del desarrollo concibió la noción de "inter-fase" entre el Estado y la sociedad civil (LONG, 2007).

8. A la inversa, como señalaremos más adelante, los movimientos sociales también ingresaron al Estado a través del nombramiento de algunos de sus líderes en puestos clave de áreas sociales. Escapa a los límites de este trabajo analizar el modo en que se "socializa" el Estado por estas vías. Remitimos a los trabajos de (NATALUCCI, 2016; PERELMITER, 2016; VÁZQUEZ, 2014). 
deramiento" de aquella suponía co-responsabilizar a las clases populares informalizadas y empobrecidas en la resolución de los problemas ligados a sus condiciones de vida. La delimitación imprecisa de la sociedad civil se resolvía, administrativamente, con la figura legal de la "asociación civil", que incluía tanto las ONG y centros de estudios que proveían técnicos y expertos, como una configuración inesperada, que tuvo lugar a nivel local, integrada por referentes barriales de todo tipo ${ }^{10}$ : partidarios, eclesiales, de movimientos sociales, que en muchos casos crearon ad hoc asociaciones civiles que les permitían acceder a los recursos públicos provenientes de los programas sociales en carácter de instancias de implementación y contraprestación a nivel local. La relación entre políticas sociales y actores territoriales no era nueva. Ya en los años sesenta los programas inspirados por las teorías de la modernización, así como el incipiente desarrollo del catolicismo de base, fueron vectores que articularon su ingreso a las zonas "marginales" con los "líderes emergentes" que allí residían. Pero a partir de los años noventa esa articulación incorporó nuevos territorios, nuevas agencias estatales y creó dinámicas más o menos rutinarias de interacción entre dirigentes locales y oficinas públicas.
Lo aprendido en los años setenta, y luego en los ochenta, con los procesos de tomas de tierra y construcción de asentamientos, en especial en el Gran Buenos Aires, fue sin duda de importancia (MERKLEN, 2005). Los dirigentes sociales y políticos barriales sabían lidiar con las diferentes agencias estatales, inclusive con las instancias represivas. Históricamente, muchos trámites y "problemas" se canalizaron a través de mediadores barriales: punteros, curas, activistas sociales. Las unidades básicas, las iglesias, las sociedades de fomento, funcionaron, así como oficinas de atención al vecino en las que se esperaba encontrar algún tipo de solución. Los mediadores entre las oficinas estatales centrales y los problemas sociales de las clases populares fueron estudiados por la literatura sobre clientelismo de los años noventa (AUYERO, 2001; MARTUCCELLI; SVAMPA, 1997). En la década de los dos mil, esta interacción se volvió incluso proyecto político de gobierno, al hacer de los movimientos sociales de matriz territorial aliados en la movilización y, en algunos casos, en la fuerza político-partidaria, proveyendo candidatos $\mathrm{y}$ hasta funcionarios a diferentes agencias estatales vinculadas con políticas urbanas y sociales (NATALUCCI, 2016; PERELMITER, 2016; VÁZQUEZ, 2014).

9. Con la creación de la Secretaría de Desarrollo Social, en 1995, se puso en marcha también el Centro Nacional de Organizaciones de la Comunidad, encargado de llevar un registro nacional de organizaciones de la sociedad civil y de establecer lazos entre ellas. Se trató del intento más sistemático de organizar al sector, establecer criterios de inclusión y categorizar a las diferentes asociaciones. Cf. (VOMMARO, 2010, cap. 3). La figura de la asociación civil existía desde el siglo XIX en el código civil nacional. Su imbricación estrecha con el ámbito de las políticas desde los años noventa puede verse, por ejemplo, en el hecho de que, en 2002, con la creación del Consejo Nacional de Coordinación de Políticas Sociales, el CENOC pasó a ser parte de esta nueva entidad. Según una encuesta realizada por el CENOC en 2004, el 35\% de las organizaciones de la sociedad civil que formaban parte de la muestra se dedicaban a tareas "sociales y humanas”, muy por encima del peso relativo de las demás áreas de especialización. Cf. (CENOC-Foro del Sector Social, 2004).

10. La noción de referente es una categoría nativa que alude a los dirigentes políticos y sociales barriales. Sus significados han sido descritos en Vommaro (2007). 
Recurrimos a nuestro trabajo de campo. Lucy, de 54 años, es dirigente del movimiento social Barrios de Pie en el barrio Ejército Argentino de la ciudad de Santiago del Estero, en el noroeste de Argentina. Ingresó a ese movimiento con la promesa de tener "planes" para poner a trabajar a "su gente" en diferentes actividades sociales que brindan servicios para los vecinos del barrio: un merendero, clases de apoyo para los estudiantes de escuelas primarias, fabricación de pan y dos huertas. El coordinador regional de Barrios de Pie, con quien se contacta periódicamente Lucy, le proveyó unos cincuenta planes del Programa de Empleo Comunitario, que suponen la realización de una "contraprestación" en tareas sociales barriales. Por entonces, el principal dirigente nacional de Barrios de Pie a nivel nacional era también funcionario del Ministerio de Desarrollo Social, a cargo de la Dirección de Asistencia Comunitaria ${ }^{11}$. A través de él, la organización tenía garantizado el acceso a programas de este tipo, que no tenían un criterio universal de atribución y que suponían una gestión local de la contraprestación ${ }^{12}$. Al mismo tiempo, la organización funcionaba como la "cara del Estado” en los barrios en los que tenía actividad; las casas de los referentes como Lucy se transformaban así en cuasi oficinas públicas, lugares de encuentro entre el Estado y la sociedad civil. Anotaciones, trámites ante los diferentes Ministerios, recepción de pedidos de inclusión en planes de otros vecinos, eran tareas en las que Lucy se especializaba y a las que dedicaba buena parte de su agenda diaria. El mismo tipo de constatación realizaron otras investigaciones en otros distritos del país. Las organizaciones que gestionan planes llegaron a tener sus propios equipos técnicos que trabajaban en anotaciones y trámites relacionados con planes sociales (MANZANO, 2013) ${ }^{13}$. y, en el caso de los programas de construcción de viviendas y urbanización de asentamientos y barrios precarios, a través de las cooperativas, funcionaron como

11. La novedad que traía esta inclusión de dirigentes sociales en el Estado no era pasada por alto por la prensa. El diario conservador La Nación, por ejemplo, titulaba: "El piquetero Jorge Ceballos es funcionario del Gobierno" (15 de julio de 2004). Paradójicamente, este dirigente de Barrios de Pie justificaba su integración al gobierno en la posibilidad de superar el "asistencialismo estatal”, definición de una forma degradada de asistencia: "la solución de fondo para los piqueteros no son los planes ni los bolsones de comida, sino formar parte de un gobierno para poder implementar políticas que modifiquen este modelo de exclusión”. Asimismo, definía sus competencias en relación al conocimiento del territorio: “Ceballos consideró que su 'principal capacidad' para ejercer el cargo para el que fue designado hace un mes en el Ministerio de Desarrollo Social reside en su conocimiento de las problemáticas de los barrios, área en la que trabaja 'desde hace más de 20 años”' (Ibid).

12. A diferencia de la Asignación Universal por Hijo (AUH), que fue implementada en 2009, y que transformó parcialmente la lógica de atribución de las políticas sociales. Se trata de una asignación monetaria que no supone contraprestación en tareas sociales, sino en términos de escolaridad y seguimiento del calendario sanitario de los menores. Desde entonces, las organizaciones barriales siguieron funcionando como oficinas de información en relación a problemas con el cobro o los controles de la contraprestación de la AUH, o como puntos de referencia para la llegada de agentes estatales a los barrios, al tiempo que mantenían otro tipo de programas sociales de gestión local. Cf. (BESANA, 2016; VOMMARO, 2015).

13. Según Manzano, "el conjunto de tareas asignadas al Equipo Técnico de Nación de la CCC se relacionaba enteramente con tareas propias de la administración de programas de empleo, entre las cuales se destacaban el ingreso de datos en sistemas informáticos, la vinculación de cada beneficiario con actividades 
oficinas de gestión de conflictos y legalidades en el barrio: a través de ellas, el Estado "se concreta localmente (y se localiza)" (FERRAUDI CURT0, 2014, p. 240).

El tipo de políticas sociales implementadas desde 2003 por los gobiernos kirchneristas fomentaron así aún más la nueva porosidad entre Estado y actores territoriales. De los planes de empleo transitorio, como el programa de cooperativas Argentina Trabaja, del Ministerio de Desarrollo Social, al Plan Fines, para la terminación de la escuela secundaria, financiado por el Ministerio de Educación, los programas tuvieron el doble objetivo de potenciar el rol de referentes barriales de las organizaciones sociales y de construir vínculos con los vecinos de esos barrios en términos de inclusión y de derechos. En tanto, por la doble vía del lugar que les otorgan las políticas y de las nuevas posiciones que ocuparon sus dirigentes y militantes en las agencias públicas, los movimientos sociales vinculados con las fuerzas de gobierno, que en algunos casos ya habían acumulado un saber hacer en la gestión de políticas durante los años ochenta y noventa, se especializaron aún más en la gestión local. En el municipio de San Isidro, por ejemplo, al ocuparse de buscar a los posibles beneficiarios del Plan Fines, el Movimiento Evita - organización social de raíz peronista - presentaba su participación en la implementación de ese programa de la siguiente manera: "Si aún no terminaste el secundario, podés contar con nosotros. El Movimiento Evita San Isidro junto al Plan Fines te dan la posibilidad de tener un título oficial del Ministerio de Educación de la Nación, con clases y horarios flexibles, cerca de tu casa y de forma gratuita. Así que acercate, nunca estuviste más cerca de lograrlo" (publicidad distribuida en la vía pública).

La literatura sobre la burocracia de calle en los Estados Unidos mostró que esos empleados “de bajo nivel” (LIPSKY, 1980, p.3) constituyen la cara del Estado ante los ciudadanos. Ellos definen la atribución de ayudas sociales ante los posibles beneficiarios. Los estudios sobre las ventanillas del Estado en los barrios populares en Francia (DUBOIS, 1999), en tanto, mostraron que los modos de existencia locales del Estado se relacionan estrechamente con la forma en que los burócratas de las ventanillas barriales desarrollan competencias para manejar la tensión entre los marcos administrativos del acceso a las ayudas públicas - a los subsidios a la vivienda, por ejemplo -, y el vínculo personal que establecen con los usuarios. Los márgenes de maniobra que las instituciones estatales dejan a esos manejos de los empleados locales constituyen el espacio de las negociaciones que aprovechan también los ciudadanos, que movilizan los recursos disponibles para contrarrestar la dominación administrativa a la que se ven sometidos. En el caso argentino, y en otros casos latinoamericanos, junto a esta burocracia de calle más clásica, existen estos actores de la "sociedad civil" que, en el mundo popular, actúan en cierta medida como agentes estatales ${ }^{14}$. Por un lado, a diferencia del caso francés, como vimos con Lucy, los espacios políticos y sociales barriales funcio-

productivas o comunitarias, la centralización de registros de asistencia de los diversos proyectos, la recepción de la nómina mensual de la liquidación del beneficio de ciento cincuenta pesos remitida por la ANSes [entidad estatal que gestiona jubilaciones y pensiones y que, desde 2002, también se ocupa de los planes sociales de alcance nacional que incluyen transferencia condicionada de dinero, como la AUH] y el reclamo por solicitudes 'rechazadas'” (MANZANO, 2013, p.185). 
nan como referencias de las oficinas estatales a nivel local. Los vecinos de los barrios conocen los múltiples espacios que existen en la zona en que habitan, y el tipo de bienes y servicios que se ofrecen en ellos, así como el tipo de relaciones que tienen éstos con las diferentes oficinas estatales - saben quiénes están cerca o lejos del gobierno de turno, pero también quiénes han desarrollado históricamente contactos en la administración pública central para resolver problemas -, y los buscan cuando los necesitan. El hecho de que la mayor parte de los espacios barriales funcione en casa de los dirigentes ayuda a encontrarlos fácilmente. Lucy, de hecho, recibía pedidos en el living de su casa. Esas relaciones no están exentas de tensiones y quejas, como sucede en las ventanillas analizadas por Dubois; en este caso, además, las reglamentaciones suelen ser más opacas para los vecinos y altamente manipuladas por los referentes. Sin embargo, aquí también las relaciones con los diferentes programas estatales se dan en el trasfondo de vínculos de más o menos largo plazo, que permiten que los vecinos conozcan a los referentes y negocien el tipo de servicios que pueden demandarles. Ya Robert Merton había identificado a las maquinarias políticas como modos de personalizar y humanizar una entidad abstracta y lejana como es la administración de las agencias estatales. Aquí, la burocracia de calle es, en cierta me- dida, una burocracia de la sociedad civil, con vínculos más o menos formales con el Estado, que van desde la movilización de conexiones organizativas con funcionarios de áreas sociales a diversas formas de contratación como operadores o animadores comunitarios y dirigentes/coordinadores de cooperativas, por ejemplo -, que hacen de los miembros de los espacios barriales de sociabilidad política agentes de las políticas sociales.

Asimismo, en los últimos años, y como parte de una política de avance de la presencia directa del Estado en los barrios populares, se organizan políticas de coordinación ministerial y hasta oficinas instaladas en conteiner, donde se pueden tramitar documentos de identidad, jubilaciones, pedidos de la AUH, etc. Esas oficinas de construcción rápida, y que parecen listas para ser desplazadas, no dejan de pasar por las mediaciones barriales, al incorporar a referentes locales que trabajan en ellas ${ }^{15}$.

Vamos a ver, ahora, el caso de la ciudad de Buenos Aires. Jonathan tiene 20 años y es militante peronista de un barrio popular de Villa Soldati - el distrito más pobre de la Ciudad. Cuando conversamos con él, trabajaba en el Programa de Inclusión a la Seguridad Social de la ANSes. Se trata de una ventanilla estatal que se instala en los barrios populares, en la que se realizan trámites vinculados con la Asignación por

14. En las últimas décadas, sociólogos y antropólogos del Estado se interesaron por el modo en que éste se imbrica con las prácticas de los actores en la vida social en general en la vida asociativa en particular, a través de sus políticas, que funcionan como dispositivos y como recursos para la acción. Cf., por ejemplo, los trabajos reunidos en Agudo Sanchiz e Estrada Saavedra (2011).

15. Lo mismo sucede, en muchos casos, con la provisión de servicios colectivos como los de agua, electricidad y gas. Recientemente, Patricio Besana ha estudiado las dinámicas políticas barriales en torno a la demanda y efectivización de servicios públicos de este tenor. Cf. (BESANA, 2016). El propio Estado organizó la construcción de una parte del tendido de cloacas y agua en barrios populares, una vez que se dejó sin efecto en 2004 la concesión del servicio en manos privadas, a través del programa "Agua más trabajo", realizado por cooperativas formadas por beneficiarios de programas sociales (MERKLEN, 2008). 
Embarazo Para Protección Social -destinada a mujeres embarazadas desde la semana 12 de gestación hasta el nacimiento o interrupción del embarazo -, la AUH, la acreditación y actualización de datos, las asignaciones familiares, así como inscripciones en programas de acceso a vivienda y a tarjetas de crédito del banco estatal. Este programa comparte muchas veces el lugar de funcionamiento con otras ventanillas de proximidad, creadas para facilitar el acceso a otras agencias estatales, como la Agencia Territorial de Acceso a la Justicia. Se instalan en espacios barriales de sociabilidad política, como centros comunitarios, o bien en Centros Integradores Comunitarios creados por el Ministerio de Desarrollo Social. "Le damos atención directa a los vecinos, para que no se coman el garrón de esperar en una oficina, que lo boludeen, yo qué sé, y les hacemos los trámites, les explicamos qué prestaciones están cobrando, cuáles pueden cobrar, tenemos conexión directa con las computadoras de la ANSes". El horario de trabajo es similar el de la mayor parte de las oficinas públicas (de 10 a 16h). El trabajo de Jonathan excede el compromiso burocrático, y expresa una gran porosidad entre el tiempo de trabajo y el tiempo de la sociabilidad barrial, como sucede en el caso de los referentes barriales que atienden a los vecino en sus casas:

hay un montón de consultas, esos puntos a veces son interministeriales, entonces yo laburo de lunes a viernes pero los sábados me llevo todas las consultas o le dejé mi número de teléfono a algún vecino, y cuando esté el de interministerial le pregunto: 'che, ¿te fijaste lo de, no sé, lo de la tenencia de los hijos de María?'. Y en el barrio también recibo preguntas y me las llevo al trabajo y ahí hablo con el abogado, por ejemplo, "che, te ha- go una pregunta', y el abogado me lo dice y yo se lo respondo a la persona, y así siempre: me fijo en la semana y el sábado cuando llevo los formularios al barrio, respondo.

Durante un tiempo, Jonathan tenía junto a sus compañeros un local partidario - unidad básica - en el barrio, donde recibía las consultas de los vecinos. Luego, por problemas con el pago del alquiler, comenzó a recibir los pedidos y consultas en su casa, o simplemente al ser abordado en encuentros ocasionales por el barrio. Aunque las oficinas en las que trabaja no se encuentran en su lugar de residencia sino en un barrio vecino, en cierta medida, él es para sus vecinos una forma de acceso a las agencias del Estado, y un modo en que el Estado puede llegar a los barrios populares.

La misma configuración es promovida “desde arriba”. Así, Mario, funcionario del área de Vivienda de la Ciudad de Buenos Aires, narra la creación de la Secretaría de Hábitat e Inclusión (SECHI) como un medio para constituir "un organismo que nucleara todas las diferentes acciones que se hacía en materia de hábitat. Y también significó plantar una bandera que no existía, que era la presencia del Estado en los barrios informales". El gobierno de la Ciudad pertenece a un partido de centro-derecha con vínculos débiles con el mundo popular. Buena parte de sus funcionarios jerárquicos provienen del mundo de los negocios (VOMMARO; MORRESI, 2014), como es el caso de Mario, por lo que tuvo que aprender a entablar conexiones - y conseguir los contactos - a nivel territorial. Para garantizar el acceso a los barrios, buscaron establecer alianzas con dirigentes locales: "tenéis que identificar líderes barriales positivos, ¿no? mesas de trabajo, nosotros trabajamos mucho en el Instituto 
de Vivienda con líderes barriales". "Líderes positivos", en este caso, significa referentes dispuestos a colaborar con un gobierno local opositor al peronismo entonces gobernante a nivel nacional. Esa búsqueda es vista como condición de posibilidad de un cierto gobierno de los barrios populares, y antes que eso como mínimo peldaño para hacer pie en ellos. "Tenéis que identificar a los líderes positivos en los cuales te podes apalancar para hacer el trabajo. Si no, la primera reacción ante la entrada del Estado es el rechazo"16. Más allá de la orientación ideológica de gobierno, la presencia estatal en los barrios populares parece ser parte de consensos de época acerca de la forma de construir estatalidad. Según Mario:

la SECHI, con sus pros y contra, sus éxitos y fracasos sembró una bandera que hoy ya nadie discute, que es la necesidad de que el Estado entre a los territorios más postergados y empiece a trabajar con la gente en la mejora de eso.

Esta presencia personalizada del Estado a través de las mediaciones locales es, muchas veces, inestable. Supone además un juego de roles por el que los referentes barriales ocupan diferentes posiciones: ora se manifiestan como representantes del Estado ante a los vecinos; ora son parte de la sociedad civil controlada por el Estado que le transfiere recursos. En ambos casos, su presencia continua en el territorio, y su inserción en regulaciones cruzadas - estatales, territoriales - otorga mayor previsibilidad a relaciones informales. Para los vecinos de los barrios populares, la alternativa es, muchas veces, una experiencia aún más conflictiva y violenta con el Estado, como la que retrata Javier Auyero (2013) en su análisis de la espera de quienes llegan a las oficinas estatales centrales o municipales sin la mediación de los referentes barriales, sea por falta de relación con ellos, o porque éstos funcionan como obstáculos a la solución de sus problemas.

El juego de roles de los referentes barriales tiene efectos en la trama social barrial. Para los beneficiarios de los programas que realizan contraprestaciones en los espacios locales de sociabilidad política, los referentes de esos espacios y las organizaciones a las que estos pertenecen son, en cierta medida, instancias de manifestación del Estado. Desde luego, los beneficiarios saben diferenciar a sus referentes del Estado, pero en la gestión cotidiana del bienestar reformulado por las políticas de combate de la pobreza ambas instancias se confunden. Los referentes anotan, gestionan, consiguen, realizan averiguaciones, y luego controlan que la contraprestación sea realizada. Aparecen, así, como burócratas estatales de la sociedad civil. En el juego de roles, sin embargo, son también voceros de esa sociedad civil ante el Estado: la buro-

16. La relación de tensión y rechazo hacia el Estado, en este caso, no sólo se relaciona con el signo político del gobierno, sino también con la experiencia negativa que tienen buena parte de las clases populares en su vínculo con las agencias estatales, en especial con el aparato represivo. En 2014, el Centro de Estudios Legales y Sociales afirmaba: "Los jóvenes que pueblan los barrios pobres, quienes deberían ser los destinatarios privilegiados de acciones que vienen procurando el crecimiento con inclusión social, están sujetos a rutinas de abuso y violencia policial y penitenciaria que erosionan las políticas de carácter inclusivo que se pretende desarrollar en esos mismos barrios. Hay zonas del Estado en las que rigen prácticas que son verdaderos obstáculos para los esfuerzos que desde otros sectores del mismo Estado se despliegan en pos de condiciones dignas de vida” (CELS, 2014, p.2). Cf. también (AUYERO, 2013). 
cracia de calle del Estado - trabajadores sociales y otros tipos de empleados de terreno -, llega a los comedores, a los merenderos y a los emprendimientos sociales para controlar a los referentes, ver el modo en que se utilizan los recursos - las raciones de comida que se entregan, por ejemplo -, y realizar informes al respecto. Los referentes reclaman ante ellos en nombre de las personas que participan de sus espacios y de sus organizaciones.

\section{2. "Llueve o truene yo vengo todos los dí- as". Programas sociales, trabajo y derechos}

La condición cuasi-estatal de la actividad realizada cotidianamente en los espacios de sociabilidad política barrial y organizada en torno a las políticas sociales se revela también en la categoría nativa de "trabajo". En efecto, todos los actores de esos espacios definen su actividad en esos términos. En el caso de los referentes, realizan un "trabajo político" para sus organizaciones de pertenencia (movilización, proselitismo) y un "trabajo social" cuasi-estatal para el barrio. Los beneficiarios de los programas, en tanto, refieren a su actividad de contraprestación como un trabajo social. En este caso, la noción de trabajo, como hemos mostrado en otra parte (VOMMARO, 2007; 2016), remite a un mundo de interacciones reguladas por ciertos principios administrativos y por ciertos principios morales que organizan las contraprestaciones, la relación entre referentes y vecinos, así como el modo en que el Estado aparece en la sociabilidad barrial. Es por eso que la noción de trabajo nos abre la puerta de cuatro dimensiones fundamentales de la vida cotidiana de los sectores populares informales que viven, en buena parte, de las actividades organizadas en torno a programas sociales. En primer lugar, per- mite repensar el topo del clientelismo, que como hemos señalado ha sido el concepto dominante para pensar las relaciones políticas establecidas a nivel local por las clases populares. El trabajo regulado por principios administrativos y morales da cuenta de vínculos atravesados por ciertas estabilizaciones de expectativas basadas en principios compartidos - principios no exentos de una dimensión de justicia, como veremos luego -, así como por conflictos en torno a su definición en ciertas situaciones que aparece, como en un vínculo laboral clásico, como violatorias de ciertas normas. De este modo, esas relaciones difieren de la visión que sobrevalora la dominación de una lógica mercantil, o bien de una lógica ideológica de deferencia ante el patrón, dominantes en las perspectivas normales de los estudios sobre clientelismo (COMBES; VOMMARO, 2015).

En segundo lugar, la actividad vista como trabajo se relaciona con una cuestión fundamental de las políticas sociales: su creciente carácter supletorio de la creación de empleo en la economía capitalista formal. En efecto, como mencionamos al comienzo de este trabajo, el diagnóstico sobre la creciente incapacidad de las economías post-industriales para producir empleos para toda su población económicamente activa es compartido. Recientemente, James Ferguson (2015) ha mostrado que las políticas de transferencia de dinero funcionan, en estas condiciones, como políticas redistributivas. En Argentina, la persistencia de una masa de trabajadores en el mercado informal llevó a repensar la relación entre ingreso y empleo. Si las políticas sociales de los años noventa buscaban mejorar la empleabilidad de las personas y se veían, en este sentido, como transitorias, las de la década siguiente comienzan a incorporar la idea de que el Estado debe financiar a un 
segmento de la población con dificultades estructurales para ingresar al mercado de trabajo formal. Eso llevó a la formulación de propuestas que van desde el fomento y financiamiento de programas que dinamicen la llamada economía popular - la formación de cooperativas de trabajo que provean servicios comunitarios, por ejemplo -, (NATALUCCI, 2016), hasta las redefiniciones de un ingreso mínimo ciudadano en términos de renta básica (LO VUOLO et al., 2011). En tiempos de escasez del trabajo formal de la sociedad industrial, el trabajo barrial parece recoger algunas de sus características. Por un lado, produce bienes materiales que se distribuyen entre los propios beneficiarios - lo cosechado en las huertas, por ejemplo -, o entre los miembros de la comunidad - los cursos de apoyo escolar que se imparten, las comidas servidas en comedores y merenderos. En otros casos, se producen bienes de apropiación colectiva, como las pequeñas obras de infraestructura que realizan las cooperativas de trabajo a nivel local (arreglos de calles, de veredas, refacciones de edificios públicos o comunitarios). Asimismo, algunos de los bienes producidos - ropa fabricada o reparada en talleres de costura, por ejemplo -, llegan a venderse en ferias informales o en actividades de microemprendedores. Por otro lado, se producen lazos sociales entre los miembros del grupo, así como bienes morales, como el orgullo de la fuerza de trabajo que algunas etnografías señalaron como cen- trales para la construcción de la identidad obrera (BEAUD; PIALOUX, 1999), y que en este caso se relaciona con el valor moral del hecho de "no quedarse quieto" (VOMMARO, 2007), que distingue a los trabajadores de los "vagos", los “aprovechadores", etc., y que forma parte de la construcción cotidiana del merecimiento que los vecinos co-producen en relación con los referentes: el "andar siempre haciendo cosas" supone una disposición al trabajo que quiere indicar una virtud que los dirigentes que dirigen los espacios locales deben reconocer. En este sentido, el valor de un plan social no sólo está asociado a la contraprestación monetaria, sino también a la cualidad moral que reconoce en sus beneficiarios ${ }^{17}$.

La contraprestación puede ser también vista como una forma de trabajo con parecidos de familia con el trabajo formal tradicional en el tipo de reglas que organizan los tiempos de las tareas, los pedidos de licencias, de suplencias, de vacaciones... Patricia, quien trabaja en una de las huertas organizadas por el espacio político de Lucy, en el barrio Ejército Argentino en Santiago del Estero, explica este aspecto de su relación con la contraprestación, y lo hace por contraste con aquellos que no merecen el reconocimiento moral del trabajo por no cumplir con las obligaciones para con las tareas cotidianas y sus regulaciones:

Sí, porque hay chicos, por ejemplo, ahí hay chicos que se ausentan mucho, y bueno. Y,

17. Esta cualidad moral es, en buena parte, impugnada por las visiones críticas de los programas sociales como subsidios para "vagos". En este contexto polémico podemos comprender los debates en torno a la adjetivación del trabajo que releva Manzano en su etnografía con movimientos sociales en el distrito de La Matanza. El trabajo en los espacios barriales puede ser "trabajo con planes" para distinguirlo del "verdadero trabajo", pero también puede ser reivindicado como "trabajo útil y genuino", o bien, en el caso de la actividad de cooperativas y micro emprendimientos, como "trabajo genuino y productivo" (MANZANO, 2013, p.197-210). 
por ejemplo, aquel chico que está por allá, tiene muchas ausencias, y le han dicho, le fueron a buscar, que le iban a sacar el plan. Y, después, dijeron que le van a dar otra oportunidad. Para mí está bien, porque ciento cincuenta [pesos, el monto de un programa social Jefas y Jefes de Hogar en 2007, entonces equivalente a unos 50 dólares], para mí es mucho. Yo no falto un día, llueve o truene, yo vengo todos los días. Si tengo que venir con mi hija, vengo. Ahora, por ejemplo, tuve que venir del barrio Independencia con mi hija, en bicicleta. Pero vengo. Ellos no. Ellos, que viven aquí, a la vuelta, ¿cómo no van a ir a trabajar? No hacen nada. ¿Porque trabajar? No hacen nada, ellos no saben valorar lo que tienen!.

Estas regulaciones del compromiso organizan el tiempo de la vida cotidiana de las personas. Al mismo tiempo, puesto que tienden a fijar obligaciones con la tarea, y no solamente con el referente, introducen un vector de despersonalización, que autonomiza, aunque sea de manera relativa, la legalidad informal de los vínculos barriales de la lógica de personalización del clientelismo político.

En tercer lugar, el trabajo es también, en cierta medida, para los beneficiarios, trabajo político, lo que remite al hecho de que los bienes y servicios producidos, así como los vínculos interpersonales que se tejen en esa actividad, participan de la existencia de una organización, de un espacio de sociabilidad política que acumula un capital colectivo, el capital territorial (ORTIZ DE
ROSAS, 2012) que supone tanto el conocimiento del espacio y de sus habitantes, como la capacidad de movilización de estos últimos ${ }^{18}$. La participación en los espacios locales de sociabilidad política realizada en torno a la contraprestación de programas sociales es así una actividad regulada por tiempos colectivos de producción que ayudan a reproducir la organización y la capacidad de movilización de los referentes territoriales: en tanto se realizan en la mayor parte de los casos en las casas de estos emprendedores políticos, reúnen a los vecinos beneficiarios - quienes van a comer, a merendar, a tomar clases de apoyo escolar -, y a los vecinos movilizados, en muchos casos categorías asumidas por las mismas personas (VOMMARO, 2016).

Por último, la concepción de la participación como trabajo se emparenta con otra dimensión de las regulaciones que se establecen en los vínculos políticos territoriales: la noción de derecho. En efecto, lejos de ser concebidos y percibidos como puros instrumentos de dominación y sujeción de los pobres, o de funcionar exclusivamente como mecanismos de chantaje político por parte de los referentes territoriales, los planes sociales - desde los planes Trabajar de los años noventa hasta los más recientes de cooperativas, de finalización de estudios, etc.-, nos permiten aprehender un modo de organización de las relaciones políticas locales que, de alguna manera, redefine el plebeyismo popular identificado por los estudios sobre el primer peronismo (JAMES, 1990), y que interioriza en los habitantes

18. Como hemos señalado en otro lado, la diferencia entre trabajo social - asistencial, productivo -, y trabajo político - de movilización de personas con fines de acumulación de poder del referente o de la organización -, son más o menos claras entre los participantes de los espacios de sociabilidad política, aunque no siempre se expresan en una distinción en el lenguaje con que se hace referencia a las actividades barriales. Cf. (VOMMARO; QUIRÓS, 2011). 
de los barrios populares la idea de que hay una cierta obligación por parte de los mediadores respecto de ellos, que los bienes de origen público que consiguen no son de su propiedad, y que la distribución es el modo más justo de existencia de esos bienes ${ }^{19}$. La noción de derecho, así, ciertamente fomentada por quienes concibieron las políticas sociales, desde los años ochenta hasta los dos milile, refleja una ambigüedad de estas políticas: negociadas localmente, y por tanto objeto de tensiones, incertidumbres (hay que "anotarse", el plan se puede “cortar”, “caer”, las esperas a veces pueden ser largas, etc.) y arbitrariedades (trámites que no llegan a buen puerto sin conocer las razones, problemas con cobros o registros que no pueden ser seguidos por los beneficiarios), son negociadas y reclamadas, en tanto recursos públicos, como "derechos de los pobres”. Una economía moral del mundo popular, asentada en esta ambigua noción de derechos, parece entonces abrir la puerta analítica de una comprensión más acabada de las complejidades de las lealtades, identificaciones y conflictos que movilizan políticamente a estas fracciones de las clases populares.

\section{Economías morales y principios de justi- cia del bienestar popular}

Los regímenes de regulación de los vínculos sociales y políticos y de la circulación bienes de origen público en los barrios populares, que definimos en relación a la categoría de trabajo, configuran una economía moral de la relación de los sectores populares con el Estado y con los espacios de sociabilidad política cuasi-estatales presentes en los barrios populares. Esta economía moral supone una cierta redefinición de la noción de derechos sociales en términos de derechos de los pobres, que recubren un abanico inespecífico de bienes y servicios - alimentos, servicios públicos, vivienda o ingresos para los hogares a partir de alguna forma de trabajo -, pero que tienen como trípode constitutivo al Estado, las organizaciones sociales y políticas territoriales y los vecinos de los barrios populares. En ocasiones, los burócratas cuasi-estatales de la sociedad civil - si a esta altura se nos permite esta definición -, son blanco de la movilización y la protesta de los vecinos de los barrios populares, quienes reclaman algún tipo de bien de origen público que

19. En otro lugar hemos señalado que una de las críticas más violentas al modo en que los dirigentes locales se relacionan con los vecinos de los barrios populares es la relacionada con el acaparamiento, es decir el recibir bienes públicos y, en lugar de distribuirlos entre los vecinos, almacenarlos o utilizarlos sólo en beneficio propio. Los rumores sobre "habitaciones llenas de mercadería" en casa de algunos dirigentes aparecieron con recurrencia en las conversaciones con los participantes de los espacios estudiados. Cf. (VOMMARO, 2007).

20. La búsqueda de hacer de los planes sociales vectores de formación de ciudadanos, y de concientizarlos de sus derechos, está presente desde el Programa Alimentario Nacional (PAN) de los años ochenta, y se mantendrá en todos los programas de lucha contra la pobreza de los años noventa. El Plan Jefas y Jefes de Hogar Desocupados, el primer programa masivo de transferencia condicionada, implementado en 2002, en contexto de crisis, en tanto, llevará la cuestión de los derechos a su misma denominación como “derecho a la inclusión”. El lenguaje de los derechos volverá a estar en el centro de las políticas públicas en los años del kirchnerismo. Cf. (RINESI, 2013). 
les es denegado. En otros momentos, son ellos quienes movilizan a los vecinos hacia las oficinas estatales para efectuar reclamos ante las autoridades municipales, provinciales o nacionales. Los criterios morales de circulación de los bienes desde el Estado hacia las clases populares constituyen entonces esos criterios de justicia que definen el modo en que los dominados conciben su derecho al bienestar.

La noción de economía moral ha conocido una revitalización en las últimas décadas, aunque en la extensión de su uso, en muchas ocasiones, perdió su dimensión política y su utilidad para pensar los modos en que los sectores subalternos negocian los términos de su subordinación (FASSIN, 2009). En efecto, en su trabajo fundador, Edward P. Thompson habló de economía moral de las multitudes para dar cuenta del modo en que se regulaba la relación entre dominantes y dominados en la Inglaterra rural del siglo XVII. Así, definió este concepto como "una visión tradicional de las normas y de las obligaciones sociales, de las funciones económicas apropiadas ocupadas por las diversas partes de la comunidad lo que tomado conjuntamente constituye la economía moral de los pobres" (THOMPSON, 1995). En esta acepción, la economía moral nos habla del modo en que se ordenan moralmente -“apropiadamente"-, relaciones sociales jerárquicas. La regulación de esos modos de subordinación remite a la movilización - implícita pero siempre explicitable -, de ciertos principios de justicia que los vuelven, en cierta medida, legítimos. Para Thompson, la economía moral es una visión "tradicional” principalmente porque está arraigada en las costumbres y la historia en común de las sociedades locales en tiempos previos a la ampliación de las fronteras producidas por el capitalismo.
De este modo, las revueltas por el aumento del precio del pan no se producían a causa del hambre, sino de la percepción de una violación de los compromisos morales que regulaban los precios - y por tanto que obligaban a los propietarios de los molinos, y a las autoridades encargadas de ordenar esa actividad -, en las configuraciones sociales que el autor estudia.

La identificación de principios de justicia como derechos a ser exigidos, por un lado, y como obligaciones, por el otro, nos permite ingresar en el mundo de la política popular munidos de herramientas que den cuenta de las regulaciones que asociamos al trabajo político y social, por un lado, y a la circulación de bienes públicos provenientes de políticas sociales, por el otro. Los programas sociales pueden convertirse, en ciertas condiciones, en algo que deben los dominantes a los dominados; al mismo tiempo, es a través del trabajo político y social que los referentes, actores dominantes en la configuración política barrial, pueden exigir a los beneficiarios compromiso con la tarea de asistencia, por un lado, y con el espacio político, por el otro. La noción de economía moral nos lleva así a pensar una economía de los dones (MAUSS, 1924-1925) entre personas con asimetría de poder definida, en cierta medida, antes de los intercambios. En nuestro caso, son los referentes territoriales, mediadores entre el centro político y el barrio - en ambos sentidos, como lo identificó claramente AUYERO (2001) recuperando los trabajos clásicos de la antropología, por ejemplo, los de F. G. Bailey (1963) sobre los brokers en India -, quienes tienen, en razón de esa posición, pero también de su lugar de "referencia” barrial, una relación asimétrica con los vecinos que “están” con ellos. El acceso a recursos de origen público que les habi- 
lita su posición de referentes - son buscados por las organizaciones políticas, como en el caso de Lucy que ya mencionamos, son movilizados por las oficinas estatales de proximidad, etc. -, sin embargo, no les asegura una posición sustentada en la pura arbitrariedad. Al contrario, diferentes formas de regulación organizan a lo largo del tiempo las relaciones - "apropiadas”, en términos morales -, entre referentes y vecinos. De hecho, impugnaciones morales a los referentes por parte de los vecinos pueden hacer perder a éstos esa posición privilegiada en los barrios populares: cuando en lugar de hacer de puente, actúan como obstáculo, pueden ser denunciados en las organizaciones de pertenencia, o bien ante burocracias de calle y oficinas estatales. En nuestro terreno, tanto en Santiago del Estero como en la ciudad de Buenos Aires, las denuncias contra referentes partidarios eran frecuentes, y tenían muchas veces como contenido el hecho de que acapararan bienes, o que los vendieran en lugar de distribuirlos entre quienes tienen derecho a ellos. En la economía moral de los pobres urbanos en tiempos de economías postindustriales, esas desviaciones pueden dar incluso lugar a denuncias ante la justicia, o ante los medios de comunicación. De esta manera podemos entender lo que Patricia cuenta acerca de los problemas que tuvo en el trabajo con otra referente, a la que acompañaba antes de “estar” con Lucy:

Y bueno trabajábamos, íbamos a los actos... $\mathrm{Y}$ encima en vez de... te sacaba plata encima porque... bueno, que hacía lotería porque era el día del niño, que hacía esto, tres pesos, que tenés que llevar algo, después las chicas le tiraban los bolsones [de alimentos], con los documentos nuestros, y llenaba la casa de bolsones, y a nadie le daba. Y después otra chica, que es la tía de la vecina de enfrente, también, mi hermana trabajaba cuidándole los chicos de ella, y mi hermana viene un día y me dice mirá, me dice, allá tienen dos piezas llenas de bolsones, llenas, hasta el techo, y eran los bolsones que retiraban durante como dos años, dos años han retirado bolsones a nombre de nosotros. Iba al interior a vender. Después se la llevaron detenida porque la denunciaron ${ }^{21}$.

En defınitiva, las relaciones cotidianas alrededor del trabajo político y social permiten acumular, conservar o ponen en riesgo el "capital moral” (WILKIS, 2013) de los referentes barriales como agentes de la justa producción y distribución de los bienes de origen público destinados a las clases populares.

Por un lado, las regulaciones entrecruzadas que mencionamos, entre el nivel de las oficinas públicas y el nivel barrial, construyen criterios de atribución de los bienes que los referentes deben seguir, o al menos cuya evitación supone todo un trabajo de ocultamiento que da cuenta de su peso normativo. La imbricación de ambos niveles se volvió más porosa desde el momento en que, a partir de 2003, los mismos actores sociales se convirtieron en agentes estatales y por tanto se familiarizaron aún más con el universo burocrático del manejo de bienes de origen público. Lucy, por ejemplo, conoce el modo en que debe llenar cartas y

21. En el barrio de Villa Soldati en el que realizamos nuestro trabajo de campo, una de las principales referentes también había sido denunciada penalmente por vender material informático destinado a cursos para los vecinos. Se decía, además, que vendía mercadería que se le daba para el comedor en su pueblo de origen, en la provincia de Salta. 
formularios (PANTALEÓN, 2005), y en caso de necesitar asesoramiento puede recurrir a los coordinadores regionales de Barrios de Pie. Cuando algún vecino relacionado con ella no cumple con los requisitos formales para acceder a un plan social, busca caminos alternativos, como otros programas sociales que no tengan el mismo tipo de normativa ${ }^{22}$. Cuando los planes tardan en "salir", consigue recursos provisorios para las personas que esperan (alimentos, trabajos ocasionales, reemplazos de quienes deben faltar por un tiempo al trabajo social de contraprestación). Ella, así como otros referentes, maniobra para lidiar, al mismo tiempo, con normas ministeriales y con criterios morales locales. La economía moral de la configuración social en la que operan se manifiesta también como un conjunto de herramientas cognitivas - un acervo de conocimiento a mano, por usar los términos de Alfred Schutz -, que orienta los términos en que se definen las relaciones entre personas de diferentes niveles (funcionarios, dirigentes, vecinos), recursos (planes, bolsones, cupos en comedores y merenderos) y actividades (trabajo político y social).

Por otro lado, el crecimiento en cantidad de programas y de beneficiarios de las políticas de lucha contra la pobreza, primero, y de las políticas sociales de inclusión, a partir de 2002, contribuyeron a reformular la noción de derecho imperante en el mundo popular y reconstruyeron ciertos criterios de justicia que tanto referentes como vecinos deben respetar. El derecho de los pobres es, en cierto modo, una reivindicación moral plebeya - es decir igualitaria, más allá de las evidentes jerarquías que imperan en esas configuraciones sociales - que opera transversalmente en las interacciones en los espacios de sociabilidad locales donde se llevan a cabo las contraprestaciones y se distribuyen bienes de origen público. Se trata, en ciertos casos, de un vector de conflicto y politización y está en el centro de las negociaciones barriales en términos de justicia. Como se trata de un derecho de desigual realización - permítasenos esta licencia inadmisible para el lenguaje jurídico -, se combina con la evaluación del acompañamiento político, y la circulación que debe hacer el referente de los recursos que recibe. Si, como señalamos, acaparar es motivo de queja por parte de los vecinos porque implica no responder a la obligación de distribución que tienen los referentes, en especial entre quienes lo acompañan, lo mismo sucede con "pedir demasiado" en el acompañamiento. Por eso es que, en muchos comedores, en los que las mujeres trabajan "ad-honorem”, a pesar de cumplir horario, éstas tienen más flexibilidad para entrar o salir antes de tiempo, y no deben conseguir reemplazo cada vez que no puede concurrir. Periódicamente, además, reciben una bolsa con mercadería - alimentos, juguetes, ropa - en forma de paga por su trabajo, aunque esta paga no está regulada por ninguna normativa estatal y hasta la contradiga (esa mercadería forma parte, muchas veces, de las raciones diarios que recibe el comedor de programas de asistencia alimentaria).

En sus investigaciones sobre asentamientos en la zona sur del conurbano, Denis

22. De hecho, el Programa de Empleo Comunitario que gestionaba cuando la visitábamos era un recurso alternativo al plan Jefas y Jefes de Hogar Desocupados, ya que tenía requisitos de acceso más laxos de admisión de beneficiarios. 
Merklen trabajó con la noción de economía moral. Allí, sostuvo: "las personas no se movilizan solamente porque tienen necesidades insatisfechas. Se movilizan a partir de un perfecto conocimiento de la coyuntura política (conscientes de lo que pueden pedir) y, a la vez, a partir de una 'moral' que les permite saber 'lo que se les debe', a qué tienen derecho" (2005, p.111). Marina Farinetti (1999), en tanto, al estudiar el estallido conocido como Santiagueñazo, que tuvo lugar en 1993, sostuvo que éste podía ser pensado como un modo de reacción popular a la ruptura de las regulaciones que regían la economía moral en Santiago del Estero, provincia en la que el sostenimiento de su población está fuertemente vinculado a la capacidad del Estado para pagar salarios. Nuestra perspectiva retoma esos análisis, y los pone al servicio de una búsqueda de los elementos que componen esa moral y que definen los derechos. Por eso nos propusimos situarnos al nivel de la interacción entre los actores. Es allí donde se puede ver el entrecruzamiento de criterios y principios que hacen a la economía moral de las clases populares informales.

¿Cómo se hace efectiva esta economía moral en la vida cotidiana? Es en las tensiones $\mathrm{y}$ discusiones entre vecinos $\mathrm{y}$ referentes, en los acuerdos sobre aquello que se puede pedir y aquello que no a un vecino, en la distinción entre aquellos que hacen trabajo político y los que sólo se dedican a las tareas asistenciales, que la economía moral se reactualiza como conjunto de principios reguladores de los vínculos políticos. Estas relaciones construyen, así, arreglos morales: las operaciones cotidianas, realizadas recursivamente pero no siempre explicitadas, aunque explicitables en caso de controversia, que producen y reproducen los principios de justicia que rigen relaciones entre referentes y vecinos, así como entre referentes barriales y referentes situados en el nivel municipal, provincial o nacional ${ }^{23}$. El costo de estos arreglos morales informales y locales es, como dijimos, la incertidumbre acerca de su sostenimiento en el tiempo. Vecinos y referentes conviven con la posibilidad de que esos derechos sean negados o suspendidos en ciertas coyunturas. Al mismo tiempo, nuevas oportunidades pueden aparecer con transformaciones en las orientaciones que gobiernan las políticas sociales. La inconstancia no implica ausencia de regulaciones. Y la precariedad no debe ser confundida con lo puramente arbitrario.

\section{Para concluir: por una perspectiva total de la política popular}

Las clases populares latinoamericanas se precarizaron en las últimas décadas. A pesar de los avances en materia social de los gobiernos progresistas y nacional-populares, la persistencia de vastos contingentes no incluidos en las dinámicas laborales del mercado formal da cuenta de que el bienestar no puede ser pensado del mismo modo que en los años de predominio de la sociedad salarial (CASTEL, 1995). En casos como el argentino, con Estados deficientes en materia de gestión social (AUYERO, 2013), la búsqueda de atajos para llegar al mundo popular a través de las mediaciones políticas y sociales existentes se revela fuente de conflictos y arbitrariedades, pero la am-

23. La noción de arreglos morales surge de un trabajo anterior, realizado junto a Julieta Quirós (VOMMAR0; QUIRÓS, 2011), en el que pensamos esta articulación entre negociación y evaluaciones morales. Luego fue reelaborada en otros textos, esta vez escritos con Hélène Combes (COMBES; VOMMARO, 2012; 2015). 
plitud y la resiliencia de este fenómeno da cuenta de su anclaje profundo en el modo en que las clases populares aprendieron a relacionarse con las agencias estatales $\mathrm{y}$ con recursos asociados al bienestar provisto por estas últimas. La consolidación de nociones de derecho que forman parte de una economía moral del acceso a bienes de origen público por parte de las fracciones informales de las clases populares muestra que no se trata de un mundo de pura dominación ni de puros intercambios instrumentales, como parece sugerir a veces la literatura sobre clientelismo (COMBES; VOMMARO, 2015; VOMMARO; QUIRÓS, 2011). A un Estado que se presenta de modo difuso en el mundo popular le corresponde una ciudadanía también difusa. El problema no es de intensidad, sino de porosidad entre situaciones e instituciones. Así como el modelo de ciudadanía que parecen tomar los estudios sobre el clientelismo ${ }^{24}$ no parece ser una perspectiva eficaz para pensar las formas efectivas de ejercicio de los derechos en esas condiciones, una visión desencarnada e instrumentalista de los vínculos políticos locales no da cuenta de las regulaciones que operan en esa existencia informal pero recursiva y recurrente de principios de justicia que organizan la economía moral del bienestar popular. En un caso, quizá, se espera demasiado de la relación entre Estado y sociedad civil; en el otro, se deja afuera demasiado al unidimensionalizar las relaciones políticas locales. Una mirada total - por utilizar los términos de Mauss -, sobre la politicidad popular necesita entonces incluir en sus análisis estas formas de existencia inesperadas - para la literatura canónica -, del Estado y la sociedad civil, así como las con- secuencias que éstas traen, en sociedades de tradición plebeyista como la Argentina, para el modo en que se ejerce la ciudadanía en territorios plagados de problemas económicos y sociales, pero no por eso menos densos en cuanto a la movilización y el activismo político.

\section{Referencias}

ACHA, 0. Sociedad civil y sociedad política durante el primer peronismo. Desarrollo Económico, v. 44, n. 174, p. 199-230, 2004.

AGUDO SANCHÍZ, A.; ESTRADA SAAVEDRA, M. (Orgs.). (Trans)Formaciones del Estado en los márgenes de Latinoamérica: Imaginarios alternativos, aparatos inacabados y espacios transnacionales. México: El Colegio de México, U. Iberoamericana, 2011.

AUYERO, J. ¿Favores por votos? Estudios sobre clientelismo político contemporáneo. Buenos Aires: Losada, 1997.

AUYER0, J. La política de los pobres: las prácticas clientelistas del peronismo. Buenos Aires: Manantial, 2001.

AUYERO, J. Pacientes del Estado. Buenos Aires: Eudeba, 2013.

BAILEY, F. G. Politics and Social Change, Orissa in 1959. Berkeley: University of California Press, 1963.

BEAUD, S.; PIALOUX, M. Retour sur la condition ouvrière: enquête aux usines Peugeot de SochauxMontbéliard. París: Fayard, 1999.

BESANA, P. Participación en asentamientos del Gran Buenos Aires (1989-2015). Tesis para obtener el Doctorado de Ciencia Política, Escuela de Política y Gobierno, Buenos Aires: UNSAM, 2016.

BEZERRA, M. 0. Em nome das "bases": política, favor e dependência pessoal. Rio de Janeiro: Relume-Dumará, 1999. 
BRIQUET, J-L. La tradition en mouvement: clientélisme et politique en Corse. París: Belin, 1997.

CASTEL, R. Les métamorphoses de la question sociale: une chronique du salariat. París: Fayard, 1995.

CELS. Las prácticas policiales violentas son contradictorias con una política de ampliación de derechos. Buenos Aires: CELS, 2014.

CENOC-Foro del Sector Social. Encuesta nacional sobre el trabajo voluntario en Argentina (20042005). Buenos Aires: CENOC-Foro del Sector Social, 2004.

COLABELLA, L. Asistentes sociales y peronistas vs. Dirigentes y referentes piqueteros en La Matanza: una reflexión sobre grados de autonomía y dependencia con el Estado. Publicar en Antropología y Ciencias Sociales, n. 11, p. 33-49, 2012.

COMBES, H. ; VOMMARO, G. Relations clientélaires ou politisation: pour dépasser certaines limites de l'étude du clientélisme. Cahiers des Amériques Latines, n. 69, p. 17-35, 2012.

COMBES, H. ; VOMMARO, G. Sociologie du clientélisme. París: La Découverte, 2015.

CORTES, R.; KESSLER, G. Políticas, ideas y expertos en la cuestión social de la Argentina democrática (1983-2012). Revista de Indias, v. 73, p. 239-264, 2013.

DEL CAMPO, H. Sindicalismo y peronismo: los comienzos de un vínculo perdurable. Buenos Aires: CLACSO, 1983.

DIAMOND, L.; MORLINO, L. The Quality of Democracy. An Overview. Journal of Democracy, v. 15, n. 4, p. 20-31, 2004.

DUBOIS, V. La vie au guichet: relation administrative et traitement de la misère. Paris: Economica, 1999.

FARINETTI, M. ¿Qué queda del "movimiento obrero"? Las formas del reclamo laboral en la nueva democracia argentina. Trabajo y Sociedad, n. 1, v. I, 1999.

FASSIN, D. Les économies morales revisitées. Annales. Histoire, Sciences Sociales, v. 64, n. 6, p. 1237-1266, 2009.
FERGUSON, J. Give a man a fish: reflections on the new politics of distribution. Durham and London: Duke University Press, 2015.

FERRAUDI CURTO, M. C. (Des)encuentros en torno a los sentidos de la política: devolución de la tesis en una organización piquetera. Nueva Antropología, v. 24, n. 75, p. 111-134, 2011.

GUPTA, A. Blurred Boundaries: The Discourse of Corruption, the Culture of Politics, and the Imagined State. American Ethnologist, v. 22, n. 2, p. 375-402, 1995.

JAMES, D. Resistencia e integración: el peronismo y la clase trabajadora argentina, 1946-1976. Buenos Aires: Sudamericana, 1990.

KESSLER, G.; DI VIRGILIO. M. La nueva pobreza urbana: dinámica global, regional y argentina en las últimas dos décadas. Revista de la CEPAL, $n$. 95, p. 31-50, 2008.

LIPSKY, M. Street-level bureaucracy: dilemmas of the individual in public services. New York: Russell Sage Foundation, 1980 (edición aniversario de 2010).

LO VUOLO, R.; RAVENTÓS, D.; YANES, P. La crisis económica, los subsidios condicionados y la renta básica. Sin Permiso: república y socialismo también para el siglo XXI, n. 10, p. 59-85, 2011.

LONG, N. Sociología del desarrollo: una perspectiva centrada en el actor. México: Ciesas/El Colegio de San Luis, 2007.

MANZANO, V. Etnografía de la gestión colectiva de políticas estatales en organizaciones de desocupados de La Matanza-Gran Buenos Aires. Runa, v. 28, p. 77-92, 2008.

MARTUCCELLI, D.; SVAMPA, M. La plaza vacía: las transformaciones del peronismo. Buenos Aires: Losada, 1997.

MAURIZIO, R.; PERROT, B.; VILLAFAÑE, S. Dinámica de la pobreza y mercado de trabajo en Argentina post-convertibilidad. Buenos Aires: Ministerio de Trabajo, Empleo y Seguridad Social y Programan de las Naciones Unidas para el Desarrollo, 2008. 
MAUSS, M. (1924-1925) Essai sur le don. París: PUF, edición de 2007.

MERKLEN, D. Vivir en los márgenes: la lógica del cazador. In: SVAMPA, M. (ed.) Desde abajo: la transformación de las identidades sociales. Buenos Aires: UNGS/Biblos, 2000, p. 81-119.

MERKLEN, D. Pobres ciudadanos. Buenos Aires: Gorla, 2005.

MERKLEN, D. L'eau et le travail. Le marché de l'eau potable, entre politiques sociales et traditions politiques, Argentine 2004-2006. Entreprises et Histoire, n. 50, p. 52-66, 2008.

NATALUCCI, A. Entre idas y vueltas. Contrapuntos sobre la intervención territorial y la construcción de estatalidades en Argentina (2009-2015). In: VOMMAR0, G. (Org.). Estado, democracia y derechos en Argentina: controversias en torno a los años kirchneristas. Buenos Aires: UNGS-CLACSO, en prensa, 2016.

OFFERLÉ, M. Le nombre de voix. Électeurs, partis et électorat socialistes à la fin du 19ème siècle en France. Actes de la recherche en sciences sociales, v. 71, n. 1, p. 5-21, 1988.

PANTALEÓN, J. Entre la carta y el formulario: política y técnica en el desarrollo social. Buenos Aires: Antropofagia-IDES, 2005.

PERELMITER, L. Burocracia plebeya: La trastienda de la asistencia social en el Estado argentino. Buenos Aires: Unsam Edita, 2016.

PLOTKIN, M. Mañana es San Perón: propaganda, rituales políticos y educación en el régimen peronista (1946-1955). Buenos Aires: Ariel, 1993.

QUIRÓS, J. El porqué de los que van: peronistas y piqueteros en el Gran Buenos Aires (una antropología de la política vivida). Buenos Aires: Antropofagia, 2011.

RIFKIN, J. The End of Work. New York: Tarcher/ Putnam, 1995.

RINESI, E. Tres décadas de democracia (1983-2013). Voces en el Fénix, año 4, n. 31, p. 6-13, 2013.
ROSANVALLON, P. La nouvelle question sociale. Repenser l'Etat providence. París: Seuil, 1995.

SEMÁN, P. Bajo continuo: exploraciones descentradas sobre cultura popular y masiva. Buenos Aires: Gorla, 2006.

THOMPSON, E. P. Costumbres en Común. Barcelona: Crítica, 1995.

VÁZQUEZ, Melina “Militar la gestión”: una aproximación a las relaciones entre activismo y trabajo en el Estado a partir de las gestiones de gobierno de Cristina Fernández de Kirchner en Argentina. Apuntes. Revista de Ciencias Sociales, v. 41, n. 74, p. 71-102, 2014.

VOMMAR0, G. “Acá no conseguís nada si no estás en política”. Los sectores populares y la participación en espacios barriales de sociabilidad política. Anuario de Estudios en Antropología Social 2006, p. 161-178, 2007.

VOMMARO, G. Regards croisés sur les rapports des classes populaires au politique en Argentine: retour sur la question du clientélisme. Tesis para la obtención del Doctorado en Sociología, París: Ecole des Hautes Etudes en Sciences Sociales, 2010.

VOMMARO, G. La participación política de los sectores populares en la Argentina reciente: transformaciones históricas y nuevos desafíos conceptuales. IN: ROFMAN, A. (Org.). Participación, políticas públicas y territorio: aportes para la construcción de una perspectiva integral. Buenos Aires: UNGS, 2000, p. 137-156.

VOMMARO, G.; MORRESI, M. Unidos y diversificados: la construcción del partido PRO en la CABA. Revista SAAP, v. 8, n. 2, p. 375-417, 2014.

VOMMARO, G.; QUIRÓS, J. "Usted vino por su propia decisión”: repensar el clientelismo en clave etnográfica. Desacatos. Revista de Antropología Social, n. 36, p. 65-84, 2011.

WILKIS, A. Las sospechas del dinero. Buenos Aires: Paidós, 2013. 
RESUMO

En el contexto económico postindustrial buena parte de las clases populares quedan fuera del mercado de trabajo formal y se producen importantes transformaciones en su relación con la actividad política, relacionadas con el modo en que las nuevas políticas sociales se insertan en las tramas socioculturales y asociativas del mundo popular. En base a materiales empíricos sobre el caso argentino, mostramos la porosidad existente entre Estado y sociedad civil popular, y la existencia de conflictivos principios morales de justicia que regulan esa interfase.

\section{PALAVRAS-CHAVE}

Política popular. Clientelismo. Políticas sociales. Estado y burocracia de calle. Economía moral. Argentina

\section{ABSTRACT}

In the post-industrial economic context, a large part of the popular classes are excluded from the formal labor market and there are important transformations in their relation to political activity, related to the way in which new social policies are inserted in the socio-cultural an associative configurations of the popular world. Based on empirical materials on the Argentine case, we show the porosity between state and "popular" civil society, and the existence of conflicting moral principles of justice that regulate that interface.

\section{KEYWORDS}

Popular politics. Clientelism. Social policies. State and Street level bureaucracy. Moral economy. Argentina. 